\title{
Measures of Complexity for Artificial Embryogeny
}

\author{
Taras Kowaliw \\ Department of Computer Science, Memorial University, \\ St. John's, Newfoundland and Labrador, Canada, A1B 3X5 \\ taras@kowaliw.ca
}

\begin{abstract}
We aim for a more rigorous discussion of "complexity" for Artificial Embryogeny. Initially, we review several existing measures from Biology and Mathematics. We argue that measures which rank complexity through a Turing machine, or measures of information contained in a genome about an environment, are not desireable here; Instead, we argue for measures which provide the environment "for free", allowing us to quantify the capacity for a genome to exploit a provided area of growth. This leads to our definition of Environmental Kolmogorov Complexity and Logical Depth, along with our introduction of novel measures of functional complexity. Next, we attempt at defining an exceptionally simple model of embryogenesis, the Terminating Cellular Automata. The described measures are computed in this context, and contrasted.
\end{abstract}

\section{Categories and Subject Descriptors}

I.2.8 [Computing Methodologies]: Artificial IntelligenceProblem Solving, Control Methods, and Search; F.1.1 [Theory of Computation]: Computation by Abstract DevicesModels of Computation

\section{General Terms}

Algorithms, Measurement, Experimentation

\section{Keywords}

Artificial Embryogeny, Developmental Systems, Complexity, Environment, Complexification, Cellular Automata

\section{INTRODUCTION}

Complexity is a topic often discussed in Artificial Embryogeny (AE) and Biology; Indeed, the notion is fundamental to several interesting open topics. Unfortunately, there does not exist any single accepted definition of the term, and many authors use it implicitly; This severely undercuts our ability to evaluate related hypotheses.

Permission to make digital or hard copies of all or part of this work for personal or classroom use is granted without fee provided that copies are not made or distributed for profit or commercial advantage and that copies bear this notice and the full citation on the first page. To copy otherwise, to republish, to post on servers or to redistribute to lists, requires prior specific permission and/or a fee.

GECCO'08, July 12-16, 2008, Atlanta, Georgia, USA.

Copyright 2008 ACM 978-1-60558-131-6/08/07 ...\$5.00.
For instance, consider the term "complexification", typically used to describe the increase in phenotypic (organismal) complexity which accompanies the growth of genomic complexity during evolution. Whether such a (causal) link exists in natural history is a much discussed topic in biology today (22,14), and has generated some interest in $\mathrm{AE}$ as well (27 2]). However, without any standard for measuring genomic or phenotypic complexity, any relevant claims are not falsifiable. This is a pity, since AE is uniquely capable of generating control data for comparison. As a second example, consider the notion of the existence of an "edge of chaos", that is, a region between orderly and chaotic growth where "interesting" algorithms lie; Evaluation of any such notion is also highly dependent upon the choice of complexity taken 9 .

This paper begins with a discussion of notions of complexity in Biology and AE. Of particular interst will be the role of the dynamic and the environment in these measures, along with biological motivations. We will not be interested in related topics such as Complexity Theory or SelfOrganization, believing this first stage to be a necessary precursor to those more ambitious topics. Current work in $\mathrm{AE}$, especially views on complexity, do not sufficiently consider the role of environment and dynamics in development; we will propose a modification of Kolmogorov complexity, along with some other measures, in an attempt to rectify this. In so doing, we will derive a definition with properties quite different from those of traditional Kolmogorov complexity; notably, we will gain computability, but lose the cross-machine applicability of traditional Kolmogorov complexity. But, we will argue, this perspective should be of greater value to practitioners.

Next, we will define perhaps the simplest model of AE possible, the Terminating Cellular Automaton (TCA). Using a one-dimensional version of TCAs on simple binary strings, we will be able to compute our defined measures (although doing so via brute force would likely be intractable in some other, less minimal model). We then contrast the measures with each other, discussing similarities and differences.

\section{REVIEW}

\subsection{Artificial Embryogeny}

Artificial Embryogeny (AE) (sometimes known as "Computational Development" or "Developmental Systems") is a sub-field of Evolutionary Computation concerned with biasing search through computation-like processes. In short, $\mathrm{AE}$ consists of the use of a non-linear dynamical system 
to map from representation to organism, inspired by, but not necessarily resembling, the mapping from genotype to phenotype in nature. There does not appear to be any accepted crisp definition; Indeed, this very conference track requests papers that deal with "the use, construction or evolution of genotype-phenotype mappings that involve either re-writing, iteration, time, or environmental interaction' $[1]$. Banzhaf and Miller phrase one of the goals of AE eloquently: "the challenge is to radically dispose of the complexity limits for the evolution of computer code, and aim at complexities heretofore only achieved by large teams of human programmers" 5].

\subsection{Cellular Automata}

Cellular Automata (CAs) originate from Ulam and von Neumann, in an attempt to describe a discrete counterpart for continuous dynamical systems 17. CAs are often used as simple models in physics, and, increasingly, as models of biological pattern formation [12, 10].

Typically CAs involve an infinite lattice of cell locations, and an arbitrarily long evolution through time. The Elementary CAs, or ECAs, are those which consist of two colours, using a neighbourhood of size three. They are often divided into four classes, originally described by Wolfram: Classes One and Two, or CAs that evolve to a stable point or period; Class Three, CAs whose behaviour is chaotic; and Class Four, CAs whose behaviour shows long-lasting structures. Unfortunately, in general, it has proven impossible to predict which class a given CA will fall into, even in a probabilistic sense; Indeed, there is no crisp definition separating Classes Three and Four, simply an agreed upon partition of the space of ECAs 3 .

\subsection{Some Measures of Complexity}

Here we review several notions of complexity, taken from both Mathematics and Biology; this is by no means an exhaustive list. Instead, we choose measures that are (a) readily defined on the space in which AE models operate, and (b) directly relevant to embryogenic growth.

Structural Complexity in organisms is an attempt to define complexity in a fashion that corresponds with our naïve conception, using the gross number of cells, cell types, organs, etc. Bell and Moorers, for instance, use the number of types of cell specialization, and show a correlation with the estimated number of cells in an organism overall. They interpret the results to indicate a greater capacity for specialization in a cooperative division of labour.Interestingly, they find that major groupings of organisms differ in complexity by size: animals have more cell types per total cell number than plants, for instance 7 .

A similar notion is that of McShae's Functional Complexity, which aims to measure the complexity of an organism by the number of functions it performs. Since an enumeration of functions is impossible directly, there have been attempts to use "parts as proxy"; That is, if one makes the assumption that parts in an organism exist because they play a functional role, then the number of parts forms a rough estimate for the number of functions which an organism carries out. Parts is a vague term, but could include the number of organs, morphological categories, etc. [23].

Shannon Entropy (sometimes Shannon Information) was developed by Shannon as a means of estimating the infor-

\footnotetext{
${ }^{1}$ From the GECCO-2007 website, sigevo.org/gecco-2007
}

mation content of a transmitted symbol 26], quite similar to notions of entropy from thermodynamics. Shannon entropy has since become a common measure of complexity in cryptography and communication theory, and also at times as a means of measuring self-organization. It is maximized for random sources and normal numbers, and low Shannon entropy is an indication of easily compressible data.

Let $X$ be a random variable taking values on alphabet $\Sigma$, and $p(x)=\operatorname{Pr}(X=x)$. The Shannon entropy function is defined as

$$
H(X)=E[I(X)]=-\sum_{x \in X} p(x) \log p(x)
$$

where $I(X)$ is the self-information, or the information contributed by a single symbol. Making the assumption that $0 \log 0=0, H$ assumes values on the range $[0, \log |\Sigma|]$.

Kolmorogov Complexity was developed independently by Kolmogorov, Chaitin and Solomonoff [15. The measure is intended to capture the notion of incompressibility of data, where we consider every knowable means of compression; This is done by considering a Universal Turing Machine, $U$, which, by the Church-Turing thesis, implements every possible algorithmic technique. The particular choice of $U$ is unimportant (from the perspective of mathematics, at least) since each can implement every other in constant time. This leads to our definition:

$$
K_{U}(x)=\min _{|p|}\left\{p \mid p \vdash_{U} x\right\}
$$

where $x$ is the data in question, $p$ is a program, and $p \vdash_{U} x$ denotes the execution of program $p$ by machine $U$ produces output $x$. Hence, we seek the shortest possible program that computes the data in question.

For both periodic and random strings (that is, strings generated by an i.i.d. variable), it is known that Shannon entropy and Kolmogorov complexity agree up to a constant [20]. Moreover, it is known that there is a link between entropy and the expectation of Kolmogorov complexity for a distribution 15. For these reasons, $H$ and $K$ are often linked - note, however, that these linkages do not imply any particular relation in the general case, and especially not if we restrict our attention to finite data. A serious drawback of Kolmogorov complexity is that it is known to be uncomputable [1], and hence, usually rejected outright.

Often the attempt is made to use compression algorithms as means of measuring the meaningful content of a pattern, having removed the obvious regularities and redundancies. Lempel-Ziv compression is a popular algorithm for the lossless compression of binary data used, for instance, in the GIF image format. The original concept was proposed by Lempel and Ziv and utilized in the specific LZW algorithm by Welch 28 .

The LZW algorithm works by creating dictionaries of necessary symbols from the base alphabet; Given a sequence in order, the system will create dictionary entries for oneand two-character sequences. As the process continues, successively longer sequences of symbols will be created - at each stage, the dictionary code for the best new symbol will be output, until the entire message has been codified. Hence, LZW is a frequency-based algorithm which removes repeated sequences. A complexity measure can be derived:

$$
L Z W(x)=\left|x^{\prime}\right|
$$

where $x^{\prime}$ is the lossless compression of string $x$. 
Compression algorithms, especially Lempel-Ziv algorithms, have been used as an approximation of Kolmogorov complexity on several occasions. This usage, in any individual case, is likely a poor choice. Although some compression algorithms have been shown to agree with $K$ is cases of high regularity and high randomness, it can easily be shown to disagree with $K$ on simple and fundamental examples.

Often, we would like to include a notion of the computational complexity required to compute a pattern; While computational complexity is a useful notion for the discussion of algorithms, it fails for the discussion of patterns, since one can always design a program that outputs some given data in time $O(1)$. One solution to this is the use of Bennet's Logical Depth 8]; In this definition, the complexity of a given piece of data is related to the running time required by the shortest algorithm which computes it. That is, given a universal Turing machine $U$ and data $x$, we define the logical depth, $D$, to be:

$$
D_{U}(x)=\tau_{U}(p *), \text { where } p * \vdash_{U} x
$$

where $\tau$ is a measure of execution time, and $p *$ is a shortestlength program computing $x$.

Note that by the criterion of logical depth, both highly ordered and random strings are not complex - since they are computed with simple 'print $x$ ' statements. Numbers such as $\pi$ or $e$, on the other hand, are considered complex, since it takes effort to print their digit expansions.

Another interesting approach comes from Adami, that of Physical Complexity, related directly to an organism's environment. Adami sets out to measure the amount of information that a given genome (or population of genomes) has about the environment; Ideally, he desires the shared Kolmogorov complexity between a sequence and a description of the environment. Since it is known that Shannon Entropy is related to the expectation of Kolmogorov complexity, it may be used as an approximation for $K$ for populations. The measure may be approximated by computing the entropies of the values at single-sites of the genome. Adami states his suspicion that biological evolution will tend towards greater complexity in time, and provides a digital experiment as illustration [1]. Unfortunately, Adami's simple model aside, the Kolmogorov complexity of the environment is likely more difficult to describe than that of an organism's genome, if for only the simple reason of relative size.

\subsection{Complexity in Artificial Embryogeny}

Unfortunately, in Artificial Embryogeny and related systems, the definition of complexity is usually left implicit. For instance, in his discussion of the generation of complex programs, Banzhaf discusses complexity only in terms of the number of components, stating simply that the generation of a program with a trillion components is "impossible" by "conventional methods" 4]; Of course, this is an approximation of truth. It is not inconceivable, for instance, to design a set-theoretic program which outputs the number one trillion using a trillion nested successor functions. In another paper, Banzhaf and Miller instead use a more Algorithmic Information-like discussion of complexity, stating

\footnotetext{
${ }^{2}$ This is easily seen by considering the compression of successively longer sequences of the expansion of a computable but normal number (one which is normal in every base is known to exist [6]), which will continue to increase in size, in contrast with its smaller $(\log n)$ Kolmogorov complexity.
}

an overall goal to be to "evolve a program whose purpose is so complex that it requires 100,000 or a million lines of code or 10,000 modules of average size 100 lines of code" ([5], emphasis added). Of course, this is also an approximation - a strict reading of this goal would require that the developmental solution also require a million lines of code to achieve the program in question, raising the question - why use an embryogenic approach?

Another approach has been taken by Hornby, who is interested in the use of developmental systems for evolutionary design. For Hornby, the term complexity should refer to our "interest and the ability to produce designs"; Hence, his notion of complexity implicitly encapsulates characteristics which directly contribute to the fitness of the generated organism. Hornby shows that the inclusion of explicit mechanisms encouraging modularity, regularity and hierarchy are positively correlated to the generation of highly fit organisms in the development of table designs 16. Hornby's usage actually implies the opposite of complexity as utilized by most physicists, seen when he demonstrates the low complexity of a random bit string.

The actual need for a developmental stage in complexification has been called into question recently by Stanley; Stanley uses the term complexification to mean that "evolution can elaborate and increase the complexity of its products by adding new refinements and divisions [new genes] during embryogenesis... this process of complexification allows evolution to discover more complex phenotypes than would be possible through optimizing a fixed set of genes." He then proposes a network of functions applied in composition as a model in which complexification may occur; These networks accept a location in space as input, then output a value by feeding through the network of functions, outputting a single value representing the specialization at the original site. Interestingly, although there does not exist any obvious developmental stage, many regularities believed significant to the canalization of space for biological design are recovered. Stanley uses a subjective evolution to generate images displaying the desired regularities - throughout the "complexity" of the image is referred to implicitly as the amount of (visually estimated) intricacy in the output imagథ 3 [27.

Some authors in AE literature are directly interested in compression-based measures; For instance, Lehre and Hartmann [19] use an LZ compression algorithm as an approximation of Kolmogorov complexity. They show that this compression corresponds to an intuitive and predictive measure of problem complexity, helping to determine the appropriateness of $\mathrm{AE}$ techniques for problem solving. A similar measure is utilized by Federici and Downing who order target patterns by "complexity" in an argument involving the inclusion of "evolutionary stages". The problem is comprised of growing images to match given patterns, each of which is given a complexity rank through a general-purpose compression algorithm, ARJ. Approximations of these patterns are then evolved through the developmental algorithm [13.

The above discussions no doubt take a more literal reading than was intended by the original authors; Indeed, Banzhaf and Miller foreshadow some of our arguments later in their paper when they note that "the complexity of the organism

\footnotetext{
${ }^{3}$ Note the potential for circularity here: subjectivity in both fitness and estimation of complexity.
} 
stems mainly from outside and has not to be provided by the genotype". However, this precise interpretation does highlight the difficulty in finding a more precise formulation, and outlines existing ambiguity.

\subsection{The Importance of Environment to Em- bryogenesis}

AE has largely been spawned from interest in the canalization of development (channelling) found in natural systems, and the hope that general and useful principles may be reproduced by computational models. Developmental systems, both natural and artificial, may be viewed as constraints of the space of all possible phenotypic configurations, and, at least in artificial cases, prevent large portions of the phenotypic space from being searched. Of course, the trade-off involves easier access to other portions of a typically large and un-evolvable phenotypic space; The same principle is described by Gould in natural systems: "Lest we begin to suspect that rigid limitation must represent the major evolutionary implication of such a constraint, I must re-emphasize the positive aspect of constraint as fruitful channelling, along lines of favourable variation that can accelerate or enhance the work of natural selection" 14.

Consider the case of the divergence of the echinoderms from their bilateral ancestors. Bilaterals are characterized by, amongst other traits, a vascular system carrying blood, a bilateral morphology; The echinoderms, on the other hand, have a radial morphology, and a transport system which carries water. Remarkably, the regulatory genes which code for these developmental traits displayed minimal changes - instead, it is their role in the dynamic of development which has altered in evolution. "The highly derived body architecture of echinoderms evolved at least in part through extensive modifications in the roles and expression domains of regulatory genes inherited from their bilateral ancestors. Even the limited number of genes and species we examined demonstrates a remarkable evolutionary flexibility" 21. It is surprising, as Gould notes, "that the evolution of differentiated and specialized Baüplane from a presumably homonomous common ancestor proceeds... by reduction and restriction, rather than by addition of genes or expansion of their domains of activity," quite unlike the mechanism specified by theories of complexification [14].

This is quite a divergence from the motivations of Physical Complexity proposed by Adami. Here, we are emphasizing the ability of the dynamic of development to utilize the environment in which it is found and adapt; This is not the same concept as the genome "having information about the environment"; Instead, the genome specifies a program which can adapt to any of a wide class of environments. It is having provided the environment "for free" that we see value in measures for practitioners of $\mathrm{AE}$; We do not seek a genome which describes our environment, but instead, a genome which can exploit an arbitrary environment. Indeed, we have evidence that such genomes exist in current AE practice [18.

\section{WHAT NOTION OF COMPLEXITY IS DESIRED?}

We aim to discuss which measures of complexity are most appropriate for AE - definable and computable descriptions which can help to formalize our intuitions, and, hope- fully, to design and guide future AE models. We are particularly interested in the perspective of a practitioner who has a model and environment already designed, as is the case with many practitioners in the field. Hence, we design methods within a given dynamic and environment, although this may remove our capacity to search between them.

Our perspective for this complexity tends to be based on a cellular granularity; That is, there appears to be a tendency to visually display cells or components, but not morphogens or resources, when discussing phenotypic complexity. This is likely influenced by biology, where time-lapse photographs of cells are sometimes used to study embryogenic phenomena (as in the study of angiogenesis by Zamir et al [29]). Further, we shall have no issue with awarding random data the highest complexity value with our measures; Random configurations require the most effort to specify, and in our opinion, thus qualify as the most complex. In the absence of a cohesive Complexity Theory, we should not make assumptions about what Self-Organization is at this particular stage.

\section{1 "Naïve" Organismal Complexity}

It is clear from the above discussion that there exists a naïve notion of phenotypic complexity which pervades our discussions, an ad hoc description based on visual inspection. The obvious analogue from Biology is Structural Complexity; Unfortunately, its use in AE directly is of dubious value. In natural systems, the inclusion of the possibility of different cell specializations requires many resources and great sophistication, whereas in most AE models, the cell types are simply pre-programmed, waiting to be activated through a single available variable.

Instead, we desire some measure that will capture the effort required, or the information contained, in the development of an organism. Actual computation of information would likely require a notion of work, which is probably impossible given our perspective; Instead, we propose using a familiar substitute, Shannon Entropy. In cases where the AE model begins with a static number of undifferentiated cells, normalized entropy may be used. If the set of all cell types is $C$, we have

$$
o C(A)=-\frac{1}{\log |C|} \sum_{i \in C} p_{i} \log p_{i}
$$

where $p_{i}$ is the frequency of cells of type $i$ in organism $A$. The use of entropy as a measure has the desirable property of additivity, corresponding to intuition.

Note the significant divergence from statistical physics, where many authors use a decrease in entropy to describe self-organization [25]. While this might be appropriate for the description of how a randomized medium transforms to an ordered one, it is not appropriate for our current perspective on $\mathrm{AE}$, which begins with a uniform mass of cells (or lack of cells altogether) that then grows and specializes. 5 .

\footnotetext{
${ }^{4}$ This assumes a simple combinatorial model of cell specification, as is typically the case. Some applications may need to substitute a different distribution function.

${ }^{5}$ Differential models of morphogenesis may seem exceptions. For instance, Merks et al note that when simulating vasculogenesis using a differential model, it is best to begin with a collection endothelian cells distributed randomly over a lattice; It is likely that application of their model decreases entropy in this case [24]. While this may be an accurate
} 


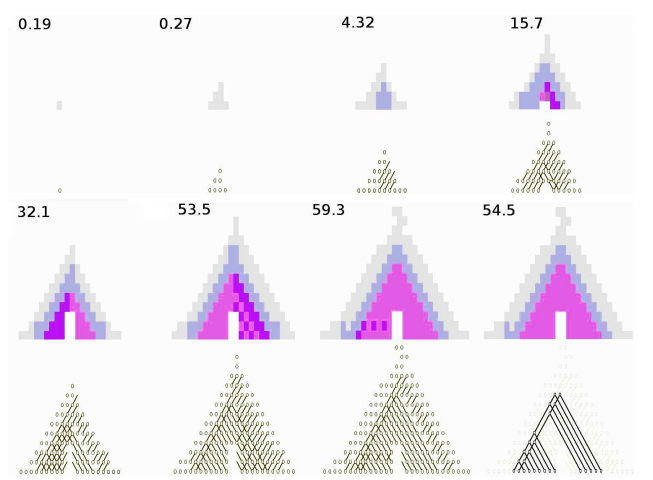

Figure 1: A Deva organism, shown at development times $0,5,10,15,20,25,30$, and 34 (termination). Inset is $o C$. Top row shows the cellular view, bottom the interpretation as a truss.

Embryogenesis, viewed at the cellular level, is an increase in disorder overall.

Figure 1 shows the computation of $o C$ for one organism from the Deva project (see 18) in various stages of development of growing a Plane Truss. One may see a slow increase in the complexity measure initially, due to undifferentiated division, then faster increases as the organism grows larger. Interestingly, the peak of $o C$ does not occur at the end, but ten time steps before, reaching a maximum of 64.3 , compared to the 54.5 value at termination. This may be a measurement of some of the self-organization which is traditionally associated with a decrease in entropy.

Unfortunately, it is impossible to compare this measure to any known relations to determine its correctness; Indeed, some biologists point out that the evidence for complexification in nature in general is inconclusive [23, which may undercut our reason for believing it exists at all; Regardless, we offer $o C$ as an improvement over implicitly defined notions, and hope that usage proves it useful.

\subsection{Genomic Complexity}

Genomic size is easily defined in AE; for variable-length representations, simple length will do. The number of genes activated during development is another, applicable to many fixed-length representations. However, we desire to extract the important parts of the genome, removing the unnecessary portions; This will help us deal with issues such as redundancy and (the genomic data formerly known as) "junk". Recall, after all, that the onion genome is more than five times the length of that of a human's!

The most direct means of capturing this is with Kolmogorov complexity. We wish to re-define this measure so as to be more applicable in describing the information in an organism's genome, and not what is given "for free" by the environment.

\subsubsection{Environmental Kolmogorov Complexity}

description, we note two issues: Firstly, the effort required to generate the random distribution is discounted, which may have been greater than the effort involved in the subsequent vasculogenesis; Secondly, it is possible that although a random distribution models reality well, that some other non-random process may also serve, hence not necessarily requiring a decrease in entropy in the first place.
Let us assume a space of genomes, $g \in G$, a space of organisms, $p \in P$, and a process, $A E$, for mapping from one to the other: $g \vdash_{A E} p$. Environment here is implicitly included in the definition of $P$. Also, let us assume that we have a measure on $G$ capturing the length of a representation, $|\cdot|: G \rightarrow \mathbf{R}$.

Given some target organism (configuration of our developmental space), $x$, we may ask the minimum size of program necessary to specify it under our scheme:

$$
e K_{A E}(x)=\min _{|g|}\left\{g \in G \mid g \vdash_{A E} x\right\}
$$

An important point about $e K$, as opposed to the original formulation of $K$, is that $e K$ need not depend on a Turing-machine 6 ; After all, we have yet to see a demonstration that Turing-completeness is required for a model of the development of living systems. Since we have a finite world imposed by the developmental (phenotypic) space, the usual trappings of uncomputability and unpredictability need not apply to $e K / 7$. Of course, the choice of embryogenic model becomes paramount, as we no longer have any guarantee of one model implementing another, as we do with Turing machines.

Note further that we may also define environmental logical depth, as an analogue of equation 3

$$
e D_{A E}(x)=\tau_{A E}(g *), \text { where } g * \vdash_{A E} x
$$

where $g *$ is a genome which minimizes $e K_{A E}$ and $\tau$ a measure of computation time.

\subsection{Functional Complexity}

For the same reasons we reject Structural Complexity for AE, we must also reject the use of "parts as proxy" for Functional Complexity - the importance of the parts has been undercut by the fact that they have been purposefully included in the model in the artificial case. However, in AE unlike Biology, we typically have the advantage that we have a raison d'être for our model: a fitness function, or a set of goals.

\subsubsection{Niche- and Funcplexity}

Here we will define a set of boolean objectives, and measure complexity in terms of which boolean objectives an organism is capable of fullfilling. A specific boolean objective, $\phi$, can be designed in an AE system in a variety of ways: by using the fitness function (" $\phi(x) \Longleftrightarrow f(x)>0.5$ "); satisfying criteria in a multi-objective optimization; meeting some specific task ("reproduce" or "survive for 100 time steps"); etc.

\footnotetext{
${ }^{6}$ One might argue that since a traditional definition of $K$ uses a universal machine $U$ capable of implementing any $\mathrm{AE}$ models in constant time, that $K$ is an appropriate measure. Here, two factors should be noted: firstly, that $U$ might be capable of finding patterns faster than our developmental system by not implementing the developmental stage; and secondly, that since our developmental system need not be Turing-complete, that $U$ might reach patterns that our system cannot.

${ }^{7}$ Even though $e K$ is computable, it is not likely tractable for any substantial model. However, it is likely that a version of Universal Codes [15] adapted to the model would be more readily computable. Or, perhaps simple random search might yield reasonable approximations, at least for the purposes of empirical evaluation of hypotheses.
} 
Then, we may consider the set of all organisms which meet objective $\phi$ :

$$
S_{\phi}=\left\{\alpha \mid \alpha \vdash_{A E} x \wedge \phi(x)\right\}
$$

Given two objectives, we may compute the set as the intersections: $S_{\{\phi, \psi\}}=S_{\phi} \cap S_{\psi}$. The complexity 8 of a niche may then be defined as:

$$
n C(\phi)=\min _{|\alpha|}\left\{\alpha \in S_{\phi}\right\}
$$

Given some organism, $x$, and a set of objectives of concern, $O=\left\{o_{1}, o_{2}, \ldots, o_{n}\right\}$, we define $F_{x}=\{o \in O \mid o(x)\} . F$ is a description of $x$ 's functionality relative to $O$. Then, we define the functional complexity as:

$$
f C(x, O)=n C\left(S_{F_{x}}\right)
$$

So, relative to objectives $O, f C$ first asks "what is the simplest agent which accomplishes everything that $x$ does, with respect to objectives $O$ ?", and returns the genomic complexity of that organism.

\section{A DOMAIN OF DISCOURSE: TCAS}

Here we define a very simple model of AE for the purpose of concrete discussion. We consider a simplified finite spacetime version of Cellular Automata, the Terminating Cellular Automata (TCAs).

Let our developmental environment, $E \subset \mathbb{Z}$, be a onedimensional toroidal line of cells of length $l$, let our alphabet be $\Sigma=\{0,1\}$, and consider our system governed by a discrete time. At time $t=0$, our environment begins with all states of colour " 0 ", save a single central cell of colour " 1 ". Our system terminates after a specified number of steps.

Then, any particular TCA, $\alpha$, is specified by a triple $(d, t, \phi)$ :

- A diameter, $0<\alpha_{d} \leq l$.

- A running time, $0 \leq \alpha_{t}<2^{l}$.

- A transition function, $\alpha_{\phi}$.

We will define the genomic size of any TCA to be its diameter, $|\alpha|=\alpha_{d}$. A rule set may be defined by enumerating all possible neighbourhoods of length $\alpha_{d}$ and specifying an output from $\Sigma$ for each 9 .

We will write that a particular TCA, $\alpha$ produces pattern $p=\left(x_{1}, \ldots, x_{l}\right)$, or

$$
\alpha \vdash_{T C A} p
$$

if running our TCA $\alpha$ for $\alpha_{t}$ steps produces lattice configuration $p$. Given some arbitrary pattern $p$, we know there exists some TCA which produces it:

THEOREM 4.1. For every pattern $p=\left(p_{1}, \ldots, p_{l}\right)$, there exists some TCA $\alpha$ such that $\alpha \vdash_{T C A} p$.

Proof. We define a TCA $\alpha$ which fits the bill. We assume $l>2$, proof is trivial otherwise. Let $\alpha_{t}=1$ and $\alpha_{d}=l$.

\footnotetext{
${ }^{8}$ We have chosen a measure ultimately linked to genomic length since we seek a measure of ease of discovery in the context of evolution; We may well have defined an alternative $n C$ as, say: $n C^{\prime}(\phi)=\min _{o C(x)}\left\{x \mid \alpha \vdash_{A E} x \wedge \alpha \in S_{\phi}\right\}$ ${ }^{9}$ Note: the Elementary Cellular Automata may be considered TCAs with $\Sigma=\{0,1\}, \alpha_{d}=3, l=\infty$ and $\alpha_{t}=\infty \forall \alpha$.
}

Consider each point on the lattice at time $t=0$ : for each location $x_{i}$, we may describe its neighbourhood, $\mu\left(x_{i}\right)$ :

$$
\mu\left(x_{i}\right)=\left(x_{i-\frac{l}{2}}, \ldots, x_{i-1}, x_{i}, x_{i+1}, \ldots, x_{i+\frac{l}{2}}\right)
$$

Since $x_{j}=1$ for exactly one $j$ (by initialization), we have $\mu\left(x_{i}\right) \neq \mu\left(x_{j}\right) \forall i \neq j$.

Now, let's define a rule set for $\alpha$ as the set $\left\{\mu\left(x_{1}\right) \rightarrow p_{1}\right.$, $\left.\ldots, \mu\left(x_{l}\right) \rightarrow p_{l}\right\}$, with all other neighbourhoods mapping to arbitrarily chosen values from $\Sigma$. It may be shown pointwise that following one time step, the lattice will contain pattern $p$.

\subsection{Measures of Complexity on TCAs}

In the case of TCAs, we may define the environmentalKolmogorov complexity as:

$$
e K_{T C A}(x)=\min _{\alpha_{d}}\left\{\alpha \mid \alpha \vdash_{T C A} x\right\}
$$

where $x$ is a binary string of length $l$. Similarly, we can define e-Logical Depth,

$$
e D_{T C A}(x)=\alpha *_{t}, \text { where } \alpha * \vdash_{T C A} x
$$

where $\alpha *$ minimizes $e K_{T C A}$.

Given some set of objectives, $O=\left\{o_{1}, \ldots, o_{k}\right\}$, we may define $n C$ and $f C$ :

$$
\begin{gathered}
n C_{T C A}(O)=\min _{|\alpha|}\left\{\alpha \mid \alpha \vdash_{T C A} x \wedge o_{1}(x) \wedge \ldots \wedge o_{k}(x)\right\} \\
f C_{T C A}(x, O)=n C(\{o \in O \mid o(x)\})
\end{gathered}
$$

\section{EXPERIMENTS WITH TCAS}

We have implemented TCAs, and used them to contrast the notions of complexity introduced above. Our domain of discourse is the set of all binary strings of length $l \in$ $\{5, \ldots, 15\} . l$ is kept reasonably small for computational reasons. For each pattern of length $l$, we have measured complexity using: Shannon Entropy $H$ (Eq. 1), EnvironmentalLogical Depth e $D_{T C A}$ (Eq. 12), the LZW algorithm (Eq. 2), and Environmental-Kolmogorov Complexity $e K_{T C A}$ (Eq. 11). In all cases, there were no patterns of $e K$ greater than five10.

Additionally, we defined a set of objectives. These were:

- $o_{\text {symm }}$ : " $x$ is symmetric", excluding the central cell for odd $l$.

- oratio2: "the number of 1's and 0's in $x$ differs by no more than 2 "

- otrans 4 : "the number of transitions from 0 to 1 (reading from left to right) is no greater than 4"

- oright0: "the rightmost cell has value 0"

- Oblack80: "the ratio of $1 \mathrm{~s}$ in $x$ is more than 0.8 "

These were divided into two sets, $O_{1}=\left\{o_{\text {symm }}, o_{\text {ratio } 2}\right.$, $\left.o_{\text {trans } 4}\right\}$ and $O_{2}=\left\{o_{\text {symm }}, o_{\text {right } 0}, o_{\text {black } 80}\right\}$. So, $O_{1}$ requires a symmetric, equally distributed pattern with few colour changes. $\mathrm{O}_{2}$ requires a pattern which is mostly 1s, with a right-most value of 0 , also symmetric. Using these sets, we were able to compute $f C$ (Eq. 13).

Using the patterns and their associated values as data points, we have computed the statistical correlation between

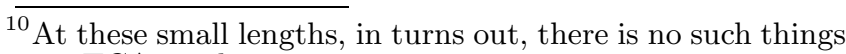
as a TCA-random string 


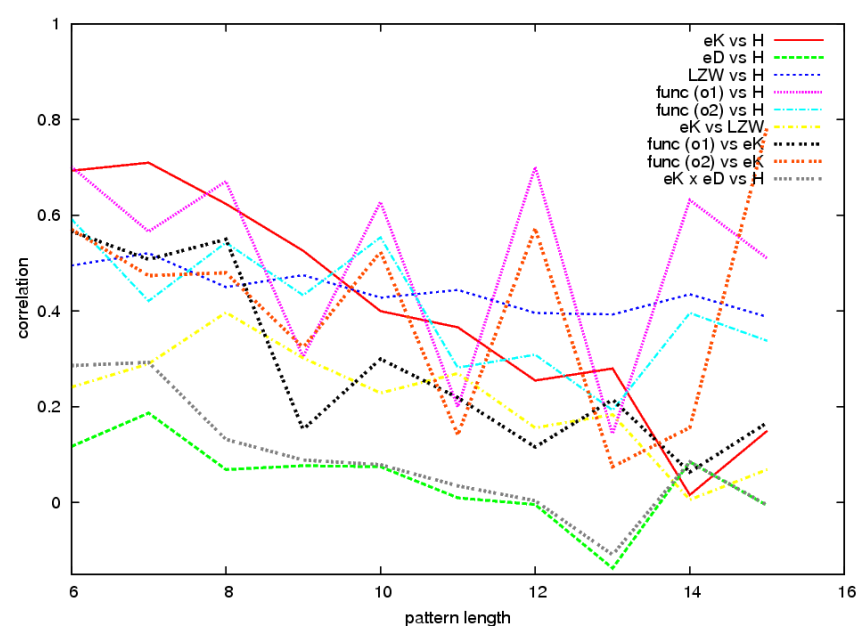

Figure 2: Correlations between complexity measures by pattern length.

the pairs (graphed in Figure 21). Further, for many of the pairs, we have computed the lines of best fit, in order to show the directness of the relation between the data.

Note firstly that all measures are generally positively correlated; This is not surprising, since all measures award very simple data low values, and all measures award random data with high values (save $e D$, the measure with the lowest correlation values). We will consider higher correlation values to indicate more agreement between measures (although that relation need not necessarily be direct).

The measure $e D$ is not well correlated to other measures at all; This is not necessarily a drawback, since the computational complexity of a growing agent is quite a unique and important concept to AE, capturing the notion of difficulty of development. Unfortunately, $e D$ did not correlate to our functional measures either, meaning that patterns with close functional value will not generally have close running times. Further, values of $e D$ varied substantially for patterns of the same functional class which had the same measures of other complexity measures, such as $e K$. Hence, we find it unlikely that $e D$ would be of practical use.

High correlation may be observed between $H$ and $f C$; This is due to the specification in the objective sets of particular proportions of cells of type 0 and 1, leading to particular values for $H$. Figure 3 compares the lines of best fit for data plots of $H$ versus $f C$ for the $l=12$ data; The relation for $O_{1}$ is far more direct, as to be expected from the criterion $o_{\text {ratio } 2}$.

Of course, there is no guarantee that patterns which satisfy some given set of objectives will have high complexity by any non-functional measure; For example, at $l=10$, the number of patterns satisfying all criteria of set $O_{1}$ was 22 , while there was only one pattern which satisfied all criteria of set $O_{2}$. All agents satisfying $O_{1}$ had an entropy of 0.971 , due to specification. Values for $e K$ and $L Z W$ were slightly higher than mean, but not significantly so. The agent satisfying $\mathrm{O}_{2}$ in fact had low values for all fields, such as $e K=2$ and $H=0.7219$, this since the pattern meeting the criterion was quite simple. A sample of the discussed patters are shown in the table below, where three patterns of length 10 satisfying $O_{1}$ are shown at the top (in order of ascending

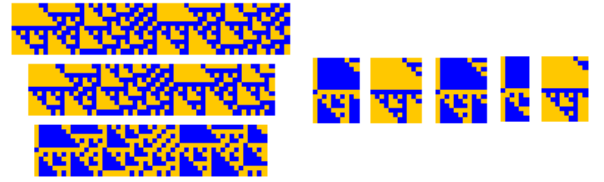

Figure 4: Two TCAs with low $e K$ generating relatively difficult to compress strings, where (left three) $l=11$ and (right five) $l=14$.

$e K)$, and one satisfying $\mathrm{O}_{2}$ is shown below:

\begin{tabular}{|c|c|c|}
\hline Pattern & $e K$ & Development \\
\hline 0001111000 & 2 & वप्र \\
\hline 0010110100 & 3 & \\
\hline 0101001010 & 4 & 놀 \\
\hline 0111111110 & 2 & 玔 \\
\hline
\end{tabular}

Note further that the correlation between $L Z W$ and $H$ is generally much higher than the correlation between either $L Z W$ and $e K$, or between $H$ and $e K$; Lines of best fit are shown in Figure 3 for $l=12$. This is not surprising, since both $H$ and $L Z W$ are frequency-based measures, while $K$ is more general. While compression algorithms may correlate to more difficult problems, and may be perfectly valid as a statistical estimate of problem complexity, there do exist forms of pattern formation which they will not measure, even in the simple case of TCAs. Indeed, the cases where the difference between $e K$ and $L Z W$ were maximized involved the growth of simple TCAs $(e K=2)$ which produced patterns which would not compress well due to a lack of regularity; Two such TCAs are shown in Figure 4

\section{CONCLUSIONS}

We have argued that it is not sufficient to use terms such as "complexity" or "complexification" naively; this, since the stated interests are not complementary. It is preferable to spell out what form of complexity one is interested in, even if vague. Several possibilities were discussed in our review, and a simple proposal was made for a naïve notion of organismal complexity.

We have further argued that Kolmogorov complexity in its classic definition is probably not desired; Instead, an environment-based Kolmogorov complexity is needed to discuss the "easiness" of specification of pattern formation in a given embryogenic context. From similar arguments, we further define some additional measures, $e D$, and functionallydefined measures.

Based on our initial experimentation with short binary strings and TCAs, we have contrasted our measures and derived some insight into their behaviour. Firstly, the various measures disagree on wide classes of data, showing clearly that they are not equivalent. Frequency-based measures, such as entropy and LZW compression do not agree well with Kolmogorov-like measures like $e K$, which do not correlate well with functional measures, such as $f C$. There does not appear to be any immediately obvious "correct" notion of complexity - for now, a practitioner must simply choose an intuitive perspective. This is likely to make evaluation of claims like the "edge of chaos" or complexification more 

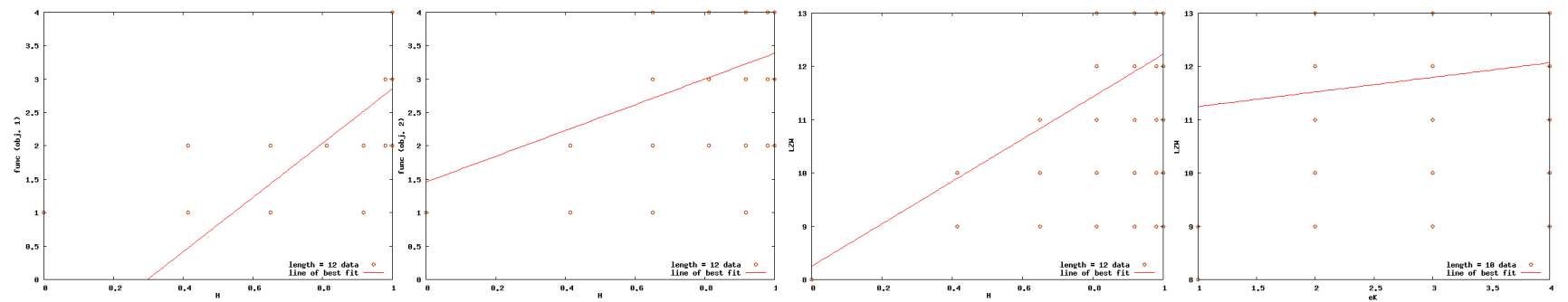

Figure 3: Best fit for: $H$ and $f C$ for (far left) $O_{1}$ and (left) $O_{2}$; (right) $H$ and $L Z W$; (far right) eK and $L Z W$.

difficult, as lacking a single accepted notion of complexity, the hypotheses lose falsifiability. To properly evaluate such claims, we must first find which class of measures, if any, has predictive value in the evolutionary process.

Further, we have determined several specific insights: (a) that environmental-Logical Depth does not display any particularly predictive properties, at least not with TCAs; (b) that our functional examples mapped to specific values for other more general measures, dictated by the objectives which the patterns met. The example serves to highlight that high fitness agents need not necessarily correspond to any particular range of values of complexity by other more general measures at all. Finally, (c) that no other measure correlated well with environmental-Kolmogorov complexity, including compression-based measures.

It is our suspicion that measures based on minimal genomic length, like environmental-Kolmogorov complexity, will prove most useful to practitioners in AE, since they readily capture factors significant to how easily a string can be found via Machine Learning. Alas, although computable, $e K$ is likely intractable for a problem of any significant phenotypic dimension. However, it is our hope that further research can find reasonable approximations, perhaps through statistical approximation or some implementation of an AEbased Universal Codes.

\section{REFERENCES}

[1] C. Adami. What is complexity? BioEssays, 24:1085-1094, 2002.

[2] L. Altenberg. Evolving better representations through selective genome growth. In IEEE CEC, 1994.

[3] T. Baldwin and S. Shelah. On the classifiability of cellular automata. Theoretical Computer Science, 230:117-129, 2000.

[4] W. Banzhaf. On evolutionary design, embodiment, and artificial regulatory networks. In F. I. et al, editor, Embodied Artificial Intelligence, LNAI. Springer-Verlag, 2004.

[5] W. Banzhaf and J. Miller. The challenge of complexity. In A. Menon, editor, Frontiers in Evolutionary Computation. Kluwer Academic, 2004.

[6] V. Becher and S. Figueira. An example of a computable absolutely normal number. Theoretical Computer Science Archive, 270(1-2):947-958, 2002.

[7] G. Bell and A. Mooers. Size and complexity among multicellular organisms. Biological Journal of the Linnean Society, 60:345-363, 1997.

[8] C. H. Bennet. On the nature and origin of complexity in discrete, homogeneous, locally interacting systems. Foundations of Physics, 16(585), 1986.

[9] N. Brodu. Practical Investigations of Complex Systems. Ph.D. thesis, Concordia U., 2007.

[10] S. Camazine, J. Deneubourg, N. Franks, J. Sneyd,
G. Theraulaz, and E. Bonabeau. Self-Organization in Biological Systems. Princeton U. Press, 2001.

[11] G. Chaitin. The Limits of Mathematics. Springer-Verlag, 1998.

[12] A. Deutsch and S. Dormann. Cellular Automaton Modelling of Biological Pattern Formation: Characterization, Applications and Analysis. Birkauser, 2005.

[13] D. Federici and K. Downing. Evolution and development of a multicellular organism: Scalability, resilience, and neutral complexification. Artificial Life, 12(3):381-409, 2006.

[14] S. J. Gould. The Structure of Evolutionary Theory. The Belknap Press of Harvard University Press, 2002.

[15] P. Grünwald and P. Vitányi. Shannon information and Kolmogorov complexity. arXiv:cs.IT/0410002, 2006.

[16] G. Hornby. Measuring, enabling and comparing modularity, regularity and hierarchy in evolutionary design. In GECCO-2005, 2005.

[17] A. Ilachinski. Cellular Automata: A Discrete Universe. World Scientific, 2001.

[18] T. Kowaliw, P. Grogono, and N. Kharma. Environment as a spatial constraint on the growth of structural form. In GECCO-200\%, 2007.

[19] P. Lehre and M. Hartmann. Development and complexity-based fitness function modifiers. In Workshop on Regeneration and Learning in Developmental Systems, 2004.

[20] M. Li and P. Vitányi. An Introduction to Kolmogorov Complexity and Its Applications. Springer, 1993.

[21] C. J. Lowe and G. A. Wray. Radical alterations in the roles of homeobox genes during echinoderm evolution. Nature, 389:718-721, 1997.

[22] D. McShae. Complexity and evolution: What everybody knows. Biology and Phil., 6:303-324, 1991.

[23] D. McShae. Functional complexity in organisms: Parts as proxies. Biology and Phil., 15:641-668, 2000.

[24] R. M. H. Merks and J. A. Glazier. A cell-centred approach to developmental biology. Physica A, 352:113-130, 2005.

[25] C. Shalizi, K. Shalizi, and R. Haslinger. Quantifying self-organization with optimal predictors. Phys. Rev. Lett., 93(118701), 2004.

[26] C. E. Shannon. A mathematical theory of communication. Bell System Technical Journal, 27:379-423 \& 623-656, 1948.

[27] K. Stanley. Exploiting regularity without development. In AAAI Fall Symposium on Developmental Systems, 2006.

[28] T. A. Welch. A technique for high-performance data compression. Computer, 17:8-19, 1984.

[29] E. Zamir, P. Rupp, and C. Little. Studying in vivo dynamics of vasculogenesis using time-lapse computational imaging. In New Frontiers in Angiogenesis. Springer-Verlag, 2006. 